\title{
Structure determination, conformational flexibility, internal dynam- ics, and chiral analysis of pulegone and its complex with water
}

\author{
Anna Krin ${ }^{a, b, c}$, Cristóbal Pérez*a,b,c ${ }^{* a b l o}$ Pinacho $^{f}$, María Mar Quesada-Moreno ${ }^{d}$, Juan Jesús López- \\ González $^{d}$, Juan Ramón Avilés-Moreno ${ }^{e}$, Susana Blanco ${ }^{f}$, Juan Carlos López ${ }^{f}$, Melanie Schnell ${ }^{* * a, b, c}$
}

\begin{abstract}
In the current work we present a detailed analysis of the chiral molecule pulegone, which is a constituent of essential oils, using broadband rotational spectroscopy. Two conformers are observed under the cold conditions of a molecular jet. We report an accurate experimentally determined structure for the lowest energy conformer. For both conformers, a characteristic splitting pattern is observed in the spectrum, resulting from the internal rotation of the two non-equivalent methyl groups situated in the isopropylidene side chain. The determined energy barriers are 1.961911(46) $\mathrm{kJ} / \mathrm{mol}$ and $6.3617(12) \mathrm{kJ} / \mathrm{mol}$ for one conformer, and $1.96094(74) \mathrm{kJ} / \mathrm{mol}$ and $6.705(44) \mathrm{kJ} / \mathrm{mol}$ for the other one. Moreover, a cluster of the lowest energy conformer with one water molecule is reported. The water molecule locks one of the methyl groups via a hydrogen bond and some secondary interactions, so that we only observe internal rotation splittings from the other methyl group with an internal rotation barrier of $2.01013(38) \mathrm{kJ} / \mathrm{mol}$. Additionally, the chirality-sensitive microwave three-wave mixing technique is applied for the differentiation between the enantiomers, which can become of further use for the analysis of essential oils.
\end{abstract}

Essential oils are widely used in medicine, pharmacology, and cosmetics. Some of the major constituents of these oils are terpenes, which are responsible for the anxiolytic ${ }^{1}$, anticonvulsive $^{2}$ or anti-inflammatory ${ }^{3}$ properties that essential oils are known for. Furthermore, many terpenes are chiral and thus may exhibit different biological functionality with respect to their enantiomers. For example, the enantiomers of the monoterpene carvone are well known to be different in odor and taste. While R-(-)-carvone smells like spearmint, its enantiomer $\mathrm{S}-(+)-$ carvone smells like caraway, indicating that our olfactory system interacts with the enantiomers of carvone in an enantioselective way. A good knowledge of the structure,

\footnotetext{
${ }^{a}$ Max-Planck Institut für Struktur und Dynamik der Materie, Hamburg, Germany.

b Deutsches Elektronen-Synchrotron, Hamburg, Germany.

${ }^{c}$ Christian-Albrechts-Universität zu Kiel, Germany.

* E-mail: cristobal.perez@desy.de

**E-mail: melanie.schnell@desy.de

d Departamento de Química Física y Analítica, Universidad de Jaén, Spain.

e Departamento de Sistemas Físicos, Químicos y Naturales, Universidad Pablo de Olavide, Sevilla, Spain .

$f$ Departamento de Química Física y Química Inorgánica, Universidad de Valladolid, Spain.
}

intramolecular dynamics, and conformational flexibility of the respective components facilitates a better understanding of the mechanisms behind these biological effects ${ }^{4}$. Such information can be obtained in the gas phase with high-resolution rotational spectroscopy.

A number of monoterpenes have been studied previously using different techniques to elucidate their molecular structure and to understand their interaction with the environment through hydrogen bonding and dispersive forces. Microwave $^{5-11}$, infrared (IR) ${ }^{12}$, Raman $^{13}$ and vibrational circular dichroism (VCD) ${ }^{14-16}$ spectroscopy represent just a few examples. Due to the recent developments in microwave spectroscopy, broadband spectra with superb signal-to-noise ratios, maintaining the high resolution of rotational spectroscopy, can be recorded in just a few hours. The high sensitivity of the technique makes it possible to observe isotopically substituted species in natural abundance and thus the changes in the molecular moments of inertia can be determined. This information can be used to determine accurate atomic positions in the molecule by solving the Kraitchman equations ${ }^{17}$.

Intramolecular dynamics, such as the internal rotation of a methyl group with respect to the remainder of the molecule, can lead to line splittings in the rotational spectra. Their analysis gives us information on the barrier height and tunneling pathways. It is also assumed that intramolecular dynamics plays important role in molecular recognition ${ }^{18}$. In addition, rotational spectroscopy allows us to characterize intra- and intermolecular interactions, as we showed recently for camphorwater ${ }^{6}$ and formamide-water clusters ${ }^{19}$, for example. Relevant questions are the preferred binding positions of water to the molecule of interest and the responsible interactions, as well as how the structure of the solutes can be affected by (micro)solvation ${ }^{20}$.

An unambiguous differentiation of the enantiomers of chiral molecules in the gas phase is possible with the newly developed microwave three-wave mixing (M3WM) technique ${ }^{21,22}$. M3WM has been applied to a number of terpenes and alcohols, including those with several stereogenic centers ${ }^{23}$, as well as for the analysis of chiral mixtures. Very recently, it was demonstrated that this technique can also be extended to achieve enantiomer-selective population transfer ${ }^{24,25}$.

Here, we report our results of a high-resolution broadband rotational spectroscopy study on the monoterpene pulegone (2-isopropylidene-5-methylcyclohexanone, Figure 1) in the 
gas phase, which provides information on the isolated species free of solvent effects. Pulegone can be found in many plants. It is a constituent of peppermint (Mentha piperita L.) and pennyroyal (Mentha pulegium) essential oils. It has a wide range of applications in food, fragrance, and pharmaceutical industry ${ }^{26}$. However, it is also known for its hepatotoxicity ${ }^{27}$. Human intoxication with pennyroyal oil can lead to gastritis, hepatic and renal failure and in severe cases even to death ${ }^{28}$. It is notable that S-(-)-pulegone is rarely found in essential oils ${ }^{29}$. This enantiomer has also been reported to have much lower toxicity in humans ${ }^{30}$.

In a recent IR-Raman-VCD study on pulegone, two conformers were determined based on quantum-chemical calculations, but only the lowest energy confomer could be observed experimentally in the liquid phase ${ }^{31}$. In the present study we extend the conformational landscape and provide a detailed characterization of pulegone both experimentally and theoretically.

\section{Experimental techniques and quantum-chemical calculations}

Pulegone was purchased as enantioenriched R-(+)- and S-(-)pulegone (97\% chemical purity and high enantiopurity, specified optical activity $\alpha_{20}^{D}=+23.5^{\circ}$ ) from Sigma-Aldrich and used without further purification. Pulegone is a liquid at room temperature and has a boiling point around $224^{\circ} \mathrm{C}$. The sample was heated to $80^{\circ} \mathrm{C}$ in a reservoir close to the valve orifice to increase the vapor pressure and then supersonically expanded into a vacuum chamber, using neon as a carrier gas (2 bar neon backing pressure, 1.5 million acquisitions averaged). For the pulegone-water cluster an additional external reservoir for water was used ( 2 bar backing pressure, 2 million acquisitions averaged).

The spectra were recorded with the Hamburg broadband chirped-pulse Fourier transform microwave (CP-FTMW) spectrometer COMPACT in the frequency range from 2-8 $\mathrm{GHz}^{32}$. The procedure can be described briefly as follows: a chirped pulse was generated with an arbitrary waveform generator (AWG), amplified with a traveling wave tube amplifier, and broadcasted into the vacuum chamber. The molecular signal was recorded as a free induction decay (FID). After the Fourier transformation of the signal from the time domain into the frequency domain, the spectrum was obtained. The detailed experimental setup is described in Ref. ${ }^{32}$. A sequence of eight $4 \mu$ s long excitation chirps was performed per gas pulse to decrease the measurement time and sample consumption, followed by the recording of the FID for $40 \mu \mathrm{s}$. For that, the fast frame option of our digital oscilloscope was employed. More details of such fast frame measurements can be found in Ref. ${ }^{33}$.
The measured rotational transitions were initially fit to an asymmetric rigid rotor Hamiltonian, using JB95 ${ }^{34}$ and PGOPHER program packages ${ }^{35}$. Refined fits were obtained using Watson's A-reduced rigid rotor Hamiltonian in the $\mathrm{I}^{\mathrm{r}}$ representation with the SPFIT program ${ }^{36}$. The internal dynamics of pulegone were analysed with the program XIAM ${ }^{37}$. XIAM uses the extended Internal Axis Method proposed by Woods ${ }^{38}$ to treat internal rotation in an asymmetric top molecule. It can handle up to three symmetric internal rotors, such as methyl groups.

The M3WM approach is described in Ref. ${ }^{10,21,39,40}$. In our experiment, a single-polarization horn was used for excitation, while a dual-polarization horn was employed for detection. M3WM relies on the fact that although the rotational constants and magnitudes of the dipole moment components $\left|\mu_{a}\right|,\left|\mu_{b}\right|$ and $\left|\mu_{c}\right|$ of a chiral molecule are the same for an enantiomeric pair, the scalar product $\left(\vec{\mu}_{a} \cdot \vec{\mu}_{b} \times \vec{\mu}_{c}\right)$ differs in sign between the enantiomers ${ }^{21}$. By using closed cycles of three rotational transitions, where each transition depends on one dipole-moment component, the so called a-, b- and c-type transitions, it is possible to distinguish between the enantiomers via a characteristic phase change of $\pi$ radians in the FID. The experiment is performed with two linearly polarized, single-frequency excitation pulses (drive and twist), which are orthogonal to each other and resonant to two different rotational transitions. In the setup used here, the drive excitation pulse was in the microwave (MW) and the twist pulse in the radio-frequency (RF) range (up to $550 \mathrm{MHz}$ ). Both excitation pulses are generated on the same AWG to ensure phase stability. The chiral molecular signal is probed on the listen transition and is recorded in a phase-dependent manner as an FID in the third mutually orthogonal direction. The recorded signal will differ by $\pi$ radians for the enantiomers due to the above-mentioned difference in sign for the product of the dipole moment components.

Quantum chemical calculations at the M06-2X/6$311++\mathrm{G}(\mathrm{d}, \mathrm{p})$ level of theory as implemented in Gaussian $09^{41}$ were performed to predict the structure, the relative energies and the dipole moment components for the most stable conformations of pulegone. Those structures were reoptimized at the $\mathrm{MP} 2 / 6-311++\mathrm{G}(\mathrm{d}, \mathrm{p})$ level of theory, for which we previously obtained reliable predictions of the rotational parameters for molecular systems similar to pulegone, such as menthone ${ }^{9}$. All conformers were tested to be real minima of the potential energy surface by checking for imaginary vibrational frequencies. In addition, several structures for pulegone-water clusters were optimized at the M06-2X/6-311++G(d,p) and B3LYP-D3BJ/6-311++G(d,p) levels of theory. 


\section{Results and Discussion}

\subsection{The pulegone monomer}

\subsubsection{Conformational flexibility}

Pulegone is a chiral molecule with the chiral center marked with an asterisk in Figure 1. The chiral center is at $\mathrm{C}$ 1, where we adopted the labeling scheme from an earlier IR-RamanVCD study on pulegone ${ }^{31}$. (Note that our labeling scheme differs from the IUPAC nomenclature, which would label the carbonyl C-atom of the cyclohexanone ring as $\mathrm{C} 1$ (our C5)). The cyclohexanone ring restricts the number of possible conformations. Using quantum chemical calculations, four conformers were identified. They are depicted in Figure 1 together with their energies relative to the lowest-energy conformer. The conformers differ in two structural features: a) the arrangement of the cyclohexanone ring, which can take on either a chair or a twist-boat configuration, and b) the orientation of the methyl group substituted to $\mathrm{C} 1$ with respect to the cyclohexanone ring (equatorial or axial). Note that in Figure 1, both R-and S-enantiomers of the Chair and the R-enantiomers of the Twist-Boat conformers are displayed.

The chair configuration with the methyl group in equatorial position, Chair 1, is the global minimum, followed by the chair configuration with the methyl group arranged axially (Chair 2). The difference between these two conformers can be better visualized by comparing the (S)-enantiomer of Chair 1 to the (R)-form of Chair 2 (Figure 1).

The calculated energy difference between the two conformers Chair 1 and Chair 2 is about $2.7 \mathrm{~kJ} / \mathrm{mol}$. A large barrier height for the interconversion of these conformers into each other is expected. This assumption is based on the fact that the energy required for ring flipping of an unsubstituted cyclohexane is on the order of $40 \mathrm{~kJ} / \mathrm{mol}$. Although this process can occur at room temperature, it will not take place under the cold conditions of the molecular jet used for our experiment. This means that both conformers should be present in the broadband rotational spectrum. The two conformers with the twist-boat configuration (Twist-Boat 1 and Twist-Boat 2) are higher in energy (11.3 and $12.2 \mathrm{~kJ} / \mathrm{mol}$, respectively) and thus should not be significantly populated.

The experimental rotational spectrum of pulegone is moderately rich, and we can clearly assign and fit the rotational spectra of two asymmetric tops. Both assigned conformers show characteristic internal rotation line splitting. The respective experimental rotational constants are given in Table 1. From a comparison with the calculated rotational values, we can identify our experimentally observed species as Chair 1 and Chair 2 , which are the energetically preferred ones.

It is noticeable that Chair 1 and Chair 2 differ clearly in their calculated rotational constants, while for Twist-Boat 1 and Twist-Boat 2 the difference is only approximately $18 \mathrm{MHz}$.
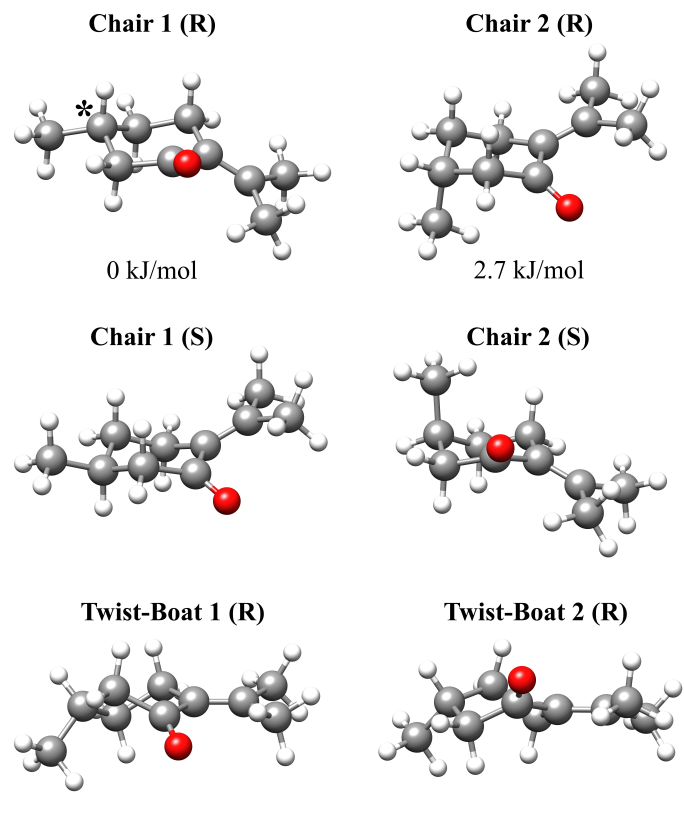

$11.3 \mathrm{~kJ} / \mathrm{mol}$

$12.2 \mathrm{~kJ} / \mathrm{mol}$

Figure 1 Structures of the four pulegone conformers, optimized at the MP2/6-311++G(d,p) level of theory, as well as their calculated relative energies. The stereogenic center is marked with an asterisk. For Chair 1 and Chair 2, both enantiomers are shown, while the Twist-Boat 1 and Twist-Boat 2 only the R-enantiomers are given.

Ray's asymmetry parameter $\kappa$ is a useful measure of the asymmetry of a molecule. Its value ranges between $\kappa=-1$ for a prolate and $\kappa=1$ for an oblate symmetric top. With $\kappa=-0.76$, pulegone is a near prolate top, with similar $\kappa$ values for all four conformers. These values are also well represented by the calculations.

An additional criterium for conformer identification are the molecular dipole-moment components $\mu_{a}, \mu_{b}$, and $\mu_{c}$ in the principal axis system. Their magnitudes determine the strengths of the different types of rotational transitions (a-, b-, and c-type) present in an experimental spectrum. For all conformers, $\mu_{b}$ is predicted to be the largest component. Indeed, the most intense lines correspond to b-type transitions. The values of $\mu_{a}$ and $\mu_{c}$ are significantly smaller. Some a-type and c-type transitions could be assigned, but with significantly lower intensity than the b-type transitions.

The conformational assignment is further supported by analysis of the structural parameters like molecular angles, determined from the fit of the torsional transitions, arising from methyl group internal rotation, as is described in more detail below.

Assuming that the expansion cools down the population of every conformer to its ground vibrational energy state, the relative populations in the supersonic jet can be obtained from 
Table 1 Comparison of experimental and calculated molecular parameters for pulegone (see Figure 1 for the labeling of the conformers). The experimental parameters of Chair 1 and 2 were determined by fitting the internal rotation of methyl tops 1 and 2 (containing C10 and C9, respectively (Figure 2)) with XIAM. The observed rotational transitions are given using the following nomenclature: $\mathrm{P}$ brunch $\triangle \mathrm{J}=-1, \mathrm{Q}$ brunch $\triangle \mathrm{J}=0, \mathrm{R}$ brunch $\triangle \mathrm{J}=+1 ; \mathrm{a}, \mathrm{b}$, and c correspond to the respective type of the rotational transitions.

\begin{tabular}{|c|c|c|c|c|c|c|}
\hline \multirow[b]{2}{*}{ Parameter } & \multicolumn{2}{|c|}{ Experimental } & \multicolumn{4}{|c|}{ MP2/6-311++G(d,p) } \\
\hline & Chair 1 & Chair 2 & Chair 1 & Chair 2 & Twist-Boat 1 & Twist-Boat 2 \\
\hline $\mathrm{A} / \mathrm{MHz}$ & $1908.49282(69)$ & $1819.9074(12)$ & 1885.42 & 1817.17 & 1974.04 & 1966.07 \\
\hline $\mathrm{B} / \mathrm{MHz}$ & $738.85952(24)$ & $816.68532(59)$ & 734.76 & 822.31 & 764.90 & 746.45 \\
\hline $\mathrm{C} / \mathrm{MHz}$ & $578.14126(23)$ & $635.94683(29)$ & 584.11 & 640.85 & 606.24 & 588.09 \\
\hline$\Delta_{J} / \mathrm{kHz}$ & $0.0330(25)$ & $0.0414(23)$ & 0.04 & 0.03 & 0.05 & 0.02 \\
\hline$\Delta_{J K} / \mathrm{kHz}$ & $-0.281(11)$ & $0.0786(58)$ & -0.32 & 0.12 & -0.22 & -0.05 \\
\hline$\Delta_{K} / \mathrm{kHz}$ & $1.231(57)$ & $-0.033(25)$ & 1.25 & -0.02 & 0.86 & 0.48 \\
\hline$\delta_{J} / \mathrm{kHz}$ & $0.00195(72)$ & & -0.001 & 0.005 & 0.01 & 0.001 \\
\hline$\delta_{K} / \mathrm{kHz}$ & & $-0.0067(12)$ & 0.06 & 0.003 & 0.01 & 0.03 \\
\hline$\kappa$ & -0.76 & -0.69 & -0.75 & -0.69 & -0.77 & -0.77 \\
\hline$V_{3}(1) / \mathrm{kJmol}^{-1}$ & $1.961911(46)$ & $1.96094(74)$ & $2.2^{\ddagger}$ & $2.2^{\ddagger}$ & - & - \\
\hline$V_{3}(2) / \mathrm{kJmol}^{-1}$ & $6.3617(12)$ & $6.705(44)$ & $5.7^{\ddagger}$ & $5.7^{\ddagger}$ & - & - \\
\hline$\measuredangle \delta(1) / \mathrm{rad}$ & $2.141735(43)$ & $2.17298(73)$ & 2.1 & 2.17 & - & - \\
\hline$\measuredangle \delta(2) / \mathrm{rad}$ & {$[2.13]^{\S}$} & $0.970(25)$ & 2.13 & 0.99 & - & - \\
\hline$\measuredangle \varepsilon(1) / \mathrm{rad}$ & $3.15223(54)$ & $3.01264(19)$ & 3.12 & 2.95 & - & - \\
\hline$\measuredangle \varepsilon(2) / \mathrm{rad}$ & $0.507(26)$ & $2.880(24)$ & 0.56 & 2.78 & - & - \\
\hline$D_{p i 2 J} *(1) / \mathrm{MHz}$ & $-0.02207(35)$ & $-0.0521(62)$ & - & - & - & - \\
\hline$D_{p i 2 K} *(1) / \mathrm{MHz}$ & $0.2705(12)$ & - & - & - & - & - \\
\hline$D_{p i 2 K} *(2) / \mathrm{MHz}$ & $0.167(86)$ & - & - & - & - & - \\
\hline$D_{p i 2-} *(1) / \mathrm{MHz}$ & $-0.02643(20)$ & $-0.0545(90)$ & - & - & - & - \\
\hline$\mu_{\mathrm{a}} / \mathrm{D}$ & ${ }^{a} R$ & ${ }^{a} R$ & 0.7 & 0.4 & -0.1 & 0.5 \\
\hline$\mu_{\mathrm{b}} / \mathrm{D}$ & ${ }^{b} R,{ }^{b} Q$ & ${ }^{b} R,{ }^{b} Q$ & -2.7 & -2.7 & -2.8 & -2.7 \\
\hline$\mu_{\mathrm{c}} / \mathrm{D}$ & ${ }^{c} R,{ }^{c} Q$ & ${ }^{c} R,{ }^{c} Q$ & -1.0 & -1.1 & 0.9 & 0.8 \\
\hline$\Delta \mathrm{E} / \mathrm{kJmol}^{-1}$ & & & 0 & 2.7 & 11.3 & 12.2 \\
\hline$\sigma^{\diamond} / \mathrm{kHz}$ & 9.09 & 6.94 & & & & \\
\hline $\mathrm{N}^{\dagger}$ & 234 & 50 & & & & \\
\hline \multicolumn{7}{|c|}{ * empirical internal rotation (overall rotation distortion operator). } \\
\hline \multicolumn{7}{|c|}{${ }^{\diamond}$ RMS deviation of the fit. } \\
\hline \multicolumn{7}{|c|}{$\dagger$ Number of fitted transitions. } \\
\hline \multicolumn{7}{|c|}{$\ddagger$ B3LYP/6-311G level of theory. } \\
\hline 8 & & & & & & \\
\hline
\end{tabular}

the Gibbs energies calculated at the conditions of the preexpansion mixture $\left(80^{\circ} \mathrm{C}, 2\right.$ bar). Using the results from $a b$-initio calculations (MP2/6-311++G(d,p) level of theory), relative populations of Chair $1 /$ Chair $2 /$ Twist-Boat 1 /TwistBoat 2 are estimated to be $1 / 0.25 / 0.02 / 0.01$. This assumption is in agreement with our experimental observation, since only Chair 1 and Chair 2 conformers were observed in the spectrum, and with the theoretical conformational analysis by Avilés-Moreno et $a l .{ }^{31}$. There they estimated the expected population for the conformer Ax-1 (corresponds to Twist-Boat 1 ) to be $1.5 \%$ at room temperature (B3LYP/cc-pVDZ level of theory). Note that in Ref. ${ }^{31}$ only the conformers Chair 1 and Twist-Boat 1 were discussed (labeled EQ-1 and AX-1), while the conformers Chair 2 and Twist-Boat 2 were not treated.

\subsubsection{Substitution structure}

A signal-to-noise $(\mathrm{S} / \mathrm{N})$ ratio of $800: 1$ for the stronger transitions of Chair 1 allowed us to observe and assign the rotational spectra of all singly substituted ${ }^{13} \mathrm{C}$ isotopologues and the ${ }^{18} \mathrm{O}$ isotopologue of the Chair 1 conformer in natural abundance. For each of the ten ${ }^{13} \mathrm{C}$ isotopologues more than 20 transitions were assigned, so that we could reliably determine the heavy-atom backbone structure. The intensity of the transitions for the ${ }^{18} \mathrm{O}$ isotopologue is in accordance with its natural abundance $(0.27 \%)$, and despite being quite weak in the spectrum, five transitions could be assigned to the ${ }^{18} \mathrm{O}$ isotopologue. Table S2 in the supplementary material summarizes the rotational parameters for all the detected isotopologues and their comparison with the parent species for the Chair 1 con- 


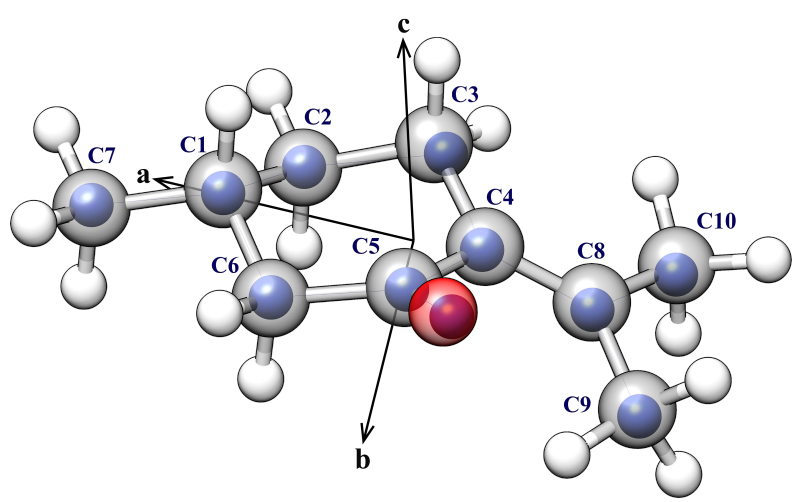

Figure 2 Experimental studies for Chair 1: $r_{0}$ (underlying structure, grey for carbon and red for oxygen) and $r_{s}$-substitution structure (blue spheres). Table S4 in the supplementary material summarizes results for bond length, and angles from $r_{0}, r_{s}$ treatments, and $a b$-initio calculations. The principal axis system is also indicated.

former of pulegone. Using the KRA program package ${ }^{17,42,43}$, we could then determine the Kraitchman substitution structure $\left(r_{s}-\right.$ structure) of Chair 1, which is displayed in Figure 2 (blue spheres).

Structure determination based on the Kraichman equations is considered as a direct way to determine the atomic positions from the changes in the moments of inertia due to isotopic substitution. However, the obtained results can be poor for atoms located close to or in plane of the principal axes. In addition, only the absolute values for the respective atomic coordinates can be determined. The sign is usually taken from $a b$ initio calculations or from chemical intuition. In the case of pulegone, C2 lies in the ab-plane, and C3 and C5 are located close to the principal axes. The calculated (MP2/6-311++G(d,p)) and the experimental $\left(r_{s}\right.$-fit) positions for the $\mathrm{C} 3$ atom differ clearly, while there is good agreement for C2 and C5 (Table S3, supplementary material).

Improved structural information can be obtained by fitting of the effective ground state structure $r_{0}$. The largest difference to the Kraitchman analysis is that it allows to exploit multi- instead of only single-isotopic substituted species. Such a $r_{0}$-fit was successfully applied in our previous studies on camphor-water clusters ${ }^{6}$, for example, producing more accurate results than the $r_{s}$-fit. A comparison between the $r_{0}$ - and the $r_{s}$-fit is given in Figure 2. Here, the underlying structure (grey for carbons, red for oxygen) represents the results from the $r_{0}$-fit, while the atom positions determined from the $r_{s}$-fit are depicted as blue shperes. As can be seen, there is a good agreement. The results (bond lengths, and angles) are summarized in Table S4 in the supplementary material.

The signal-to-noise ratio was not sufficient (approx. 30:1 for the strongest transitions) to determine any isotopologues for the Chair 2 conformer.

\subsubsection{Internal rotation}

The rotational spectrum of pulegone shows clear substructure due to intramolecular dynamics. We identify multiplets with components spaced over several MHz (Figure 3).

Pulegone has three methyl tops (Figure 2), labeled top 1 (containing C10), top 2 (containing C9), and top 3 (containing $\mathrm{C} 7$ and being attached to the chiral center of the molecule, C1). Methyl tops 1 and 2 are substituted to the same $\mathrm{sp}^{2}$ hybridized carbon atom $\mathrm{C} 8$, so that a rather low barrier to internal rotation can be expected. Indeed, the calculated barriers are 2.2 and $5.7 \mathrm{~kJ} / \mathrm{mol}$ for top 1 and top 2, respectively (B3LYP/6-311G level of theory). For top 3, the calculated barrier is about $12 \mathrm{~kJ} / \mathrm{mol}$ (B3LYP/6-311G level of theory), which is too high to give rise to detectable line splittings for the frequency resolution of approximately $25 \mathrm{kHz}$ of the COMPACT spectrometer $^{9,44}$

The internal rotation of the methyl tops 1 and 2 gives rise to the fine structure illustrated in Figure 3. Multiplets with components spanning several $\mathrm{MHz}$ were identified. The multiplets were analyzed and fitted using the program XIAM. First, a subset containing 97 lines was fitted separately to a rigidrotor Hamiltonian with standard deviation of $7.53 \mathrm{kHz}$ (Table $\mathrm{S} 1$, supplementary material). This fit was then used to predict the splittings for the strongest b-type transitions of the other internal rotation components of Chair 1 using the calculated internal rotation barriers. The coupling scheme is depicted in Figure S2, supplementary material. Within the local mode picture, the multiplets are labeled as AA, AE, EA and EE and EE*. In a step-by-step procedure, a total of 234 lines were assigned with a standard deviation of $9.09 \mathrm{kHz}$ (Table 1).

For Chair 2, the assignment of the torsional transitions was more challenging due to the lower intensity. The values for the experimentally determined internal rotation barriers of Chair 1 were used as a starting point. Overall, 50 lines could be assigned and fitted for Chair 2 with standard deviation of 6.94 $\mathrm{kHz}$ (Table 1). The complete line list of all assigned transitions for Chair 1 and 2 is provided in the supplementary material. The multiplet structure due to the internal dynamics of Chair 2 is presented in Figure S3 (supplementary material).

The internal rotations of the methyl groups of Chair 1 are hindered by only low barriers of $1.961911(46) \mathrm{kJ} / \mathrm{mol}$ for top 1 , which is pointing away from the carbonyl group, and $6.3617(12) \mathrm{kJ} / \mathrm{mol}$ for top 2, which interacts with the carbonyl group, thus reulting in the higher barrier. For Chair 2, extremely similar barrier heights were determined (1.96094(74) $\mathrm{kJ} / \mathrm{mol}$ for top 1 and $6.705(44) \mathrm{kJ} / \mathrm{mol}$ for top 2). Note that for both Chair 1 and Chair 2 the internal rotation barrier was determined with higher accuracy for methyl top 1 than for top 2. This is mainly due to the fact that AA and AE splittings could not be resolved in our experiment predicted frequency difference less than $20 \mathrm{kHz}$ ). For an accurate determination 



Figure 3 Part of the broadband rotational spectrum (1.5 million acquisitions and using neon as carrier gas) of the pulegone monomer. The upper part gives an overview, while the lower three sections are zoom-ins to individual rotational transitions to illustrate the characteristic multiplet structure due to internal dynamics. In all parts, the upper traces (black) are the experimentally obtained spectra. The lower (red) traces are stick spectra (not taking into account specific intensity distributions) based on simulations using the fitted molecular constants for Chair 1. The labeling of the rotational transitions follows the $J_{K_{a} K_{c}}$ scheme.

of the internal rotation barrier for methyl top 2 these splittings need to be taken into account. The internal rotation barrier for methyl top 1 depends more strongly on EA, EE and EE* splittting, which are well resolved in the spectrum (Figure 3).

It is interesting to compare the internal rotation of pulegone to that of acetone. Since both molecules have two methyl groups attached to an $\mathrm{sp}^{2}$ hybridized carbon atom, the patterns for the internal rotation splittings are expected to be similar. The two methyl groups of acetone are equivalent, and their internal motion gives rise to quartets ${ }^{45}$. In pulegone, the two methyl tops are non-equivalent due to their different chemical environment, resulting in a more complex splitting pattern (Figure 3). The experimental $V_{3}$ barrier for acetone is $3.18327(59) \mathrm{kJ} / \mathrm{mol}^{45}$, which is an intermediate value between the two non-equivalent methyl tops of pulegone. As it could be expected, the barrier for acetone is closer to the barrier of top 1 in pulegone, which is not affected by any interaction with neighboring groups.

An additional aspect in this context is the structural information gained from the internal rotation fits. As mentioned previously, the information about the angles between the in- ternal rotation axis and the respective principal axes can be helpful for identifying molecular conformers. Here, the angle $\varepsilon(2)$, which is defined as the angle between the principal axis $\mathrm{b}$ and the projection of the internal rotation axis in the principal axis system ${ }^{46}$, is significantly different for Chair 1 and 2 (0.507(26) rad Chair 1, 2.880(24) rad Chair 2, Table 1). By comparing the values for $\varepsilon(2)$ from the XIAM fit to the $a b$ initio calculations (0.56 rad Chair 1 and 2.78 rad Chair 2, Table $1)$, the previous identification of the two conformers also is supported.

\subsubsection{Enantiomer differentiation using M3WM}

M3WM experiments were carried out with enantioenriched samples of pulegone. This allows an unambiguous distinction between the enantiomers, which can then be used for chiral analysis of complex chiral mixtures involving pulegone, such as essential oils. For M3WM, closed cycles consisting of a-, b-, and c-type rotational transitions have to be identified and optimized first, as described in more detail in Ref. ${ }^{10}$. For pulegone, two M3WM cycles were optimized and successfully ap- 

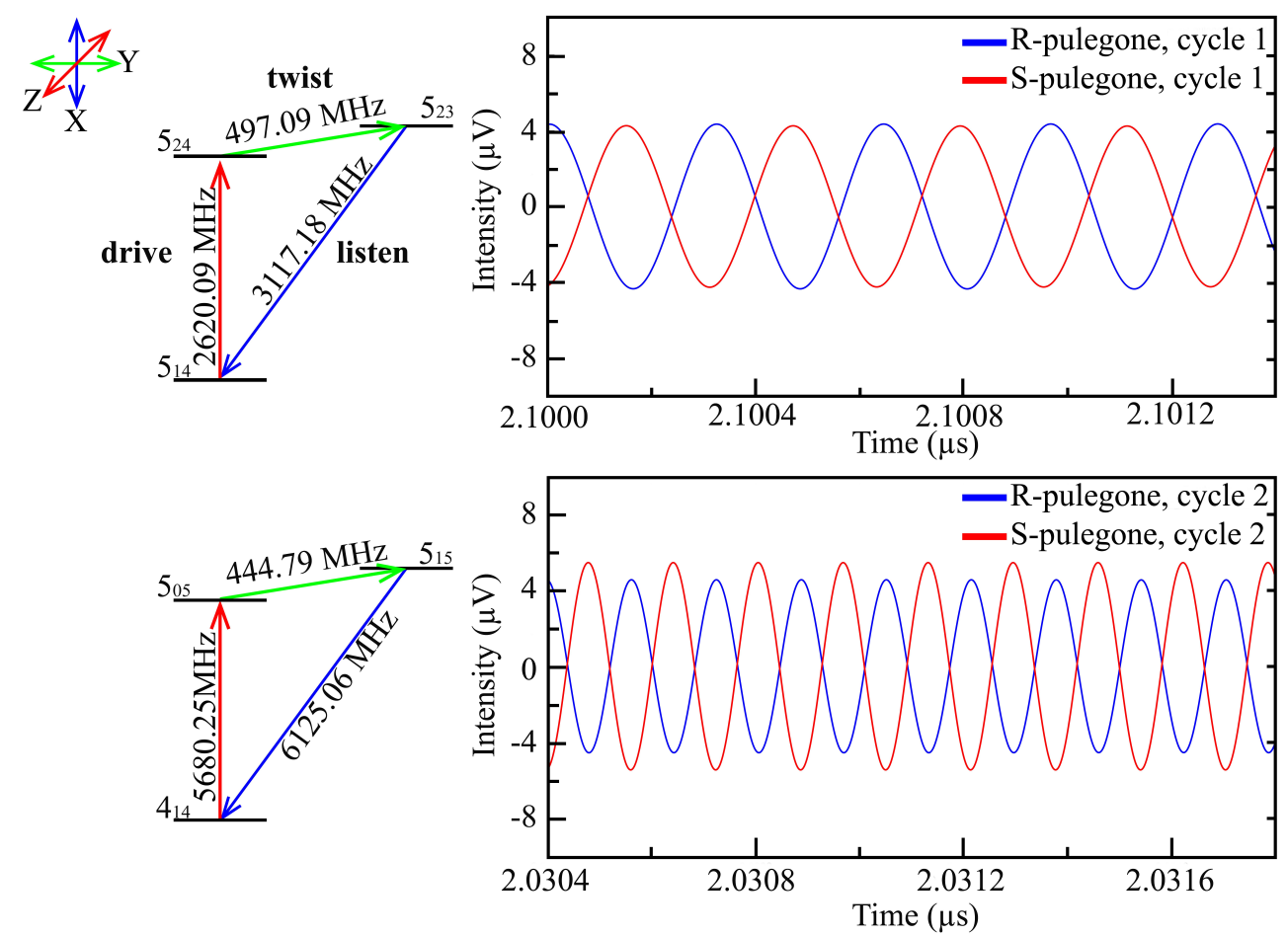

Figure 4 Two M3WM cycles for the pulegone conformer Chair 1. The nomenclature for labeling the respective rotational transitions is $J_{K_{a}} K_{c}$. The linearly polarized MW pulse (drive, defined to be along the $\mathrm{Z}$ axis in the laboratory space) and radio frequency (RF) pulse (twist, defined along the $\mathrm{Y}$ axis) are the two orthogonal excitation pulses. The molecular M3WM response (listen) in form of an FID is recorded in X direction.

plied for conformer Chair 1 (Figure 4). The transitions in these cycles only involve states of AA symmetry with respect to internal dynamics. Cycle 1 solely consists of Q-branch transitions that obey the selection rule $\Delta \mathrm{J}=0$, which is an interesting aspect to note.

The M3WM cycles were optimized with respect to the durations of the two excitation pulses, the drive and the twist. It has been worked out before that optimal M3WM signal is obtained for drive pulse durations fulfilling $\pi / 2$ Rabi conditions, thus inducing maximum coherence between the two states involved. The twist pulse durations should fulfill $\pi$ pulse conditions ${ }^{47}$. The $\pi$ twist pulse transfers coherence from the $5_{24} \leftarrow 5_{14}$ (induced by the $\pi / 2$ drive pulse) to the $5_{14} \leftarrow 5_{23}$, which is then recorded (Figure 4, cycle 1).

For optimizing the duration of the drive pulses, the transitions (cycle 1: c-type transition $5_{24} \leftarrow 5_{14}$ at $2620.09 \mathrm{MHz}$; cycle 2: b-type transition $5_{05} \leftarrow 4_{14}$ at $5680.25 \mathrm{MHz}$ ) were directly excited with increasing duration, and their signal amplitudes were monitored. For both transitions, the optimal pulse duration was $50 \mathrm{~ns}$ to achieve maximum signal and thus $\pi / 2$ pulse conditions (Figure S1, supplementary material).

In our current setup, the twist transitions are in the radiofre- quency (RF) range between 50-550 $\mathrm{MHz}$ and can not be directly monitored. For optimizing their pulse durations to $\pi$ condition, a double-resonance approach was employed ${ }^{48}$. The amplitude of the respective listen transition was monitored as a function of the twist pulse duration. The optimal twist pulse duration was $1600 \mathrm{~ns}$ for $5_{23} \leftarrow 5_{24}$ at $497.09 \mathrm{MHz}$ (a-type transition) for cycle 1 , and $300 \mathrm{~ns}$ for $5_{15} \leftarrow 5_{05}$ (c-type transition) at $444.79 \mathrm{MHz}$ for cycle 2. This clear difference in optimal pulse duration mainly arises from the properties of the capacitor plates that we use to broadcast the RF twist excitation pulses. Some frequencies are more strongly supported due to resonances than others.

Using M3WM, enantiomer differentiation is achieved via a characteristic, unambiguous phase difference observed in the time domain (FID) for the listen transition of the M3WM cycle. It arises from the sign difference in the overall product of the dipole-moment components. The results for pulegone are depicted in Figure 4. The phase shift of $\pi$ rad is clearly visible for both cycles. The M3WM cycles worked out in this study will be used in subsequent experiments on pulegone in essential oils. Comparison of the FID phases for the enantioenriched sample to the ones in the oil will allow us to determine 

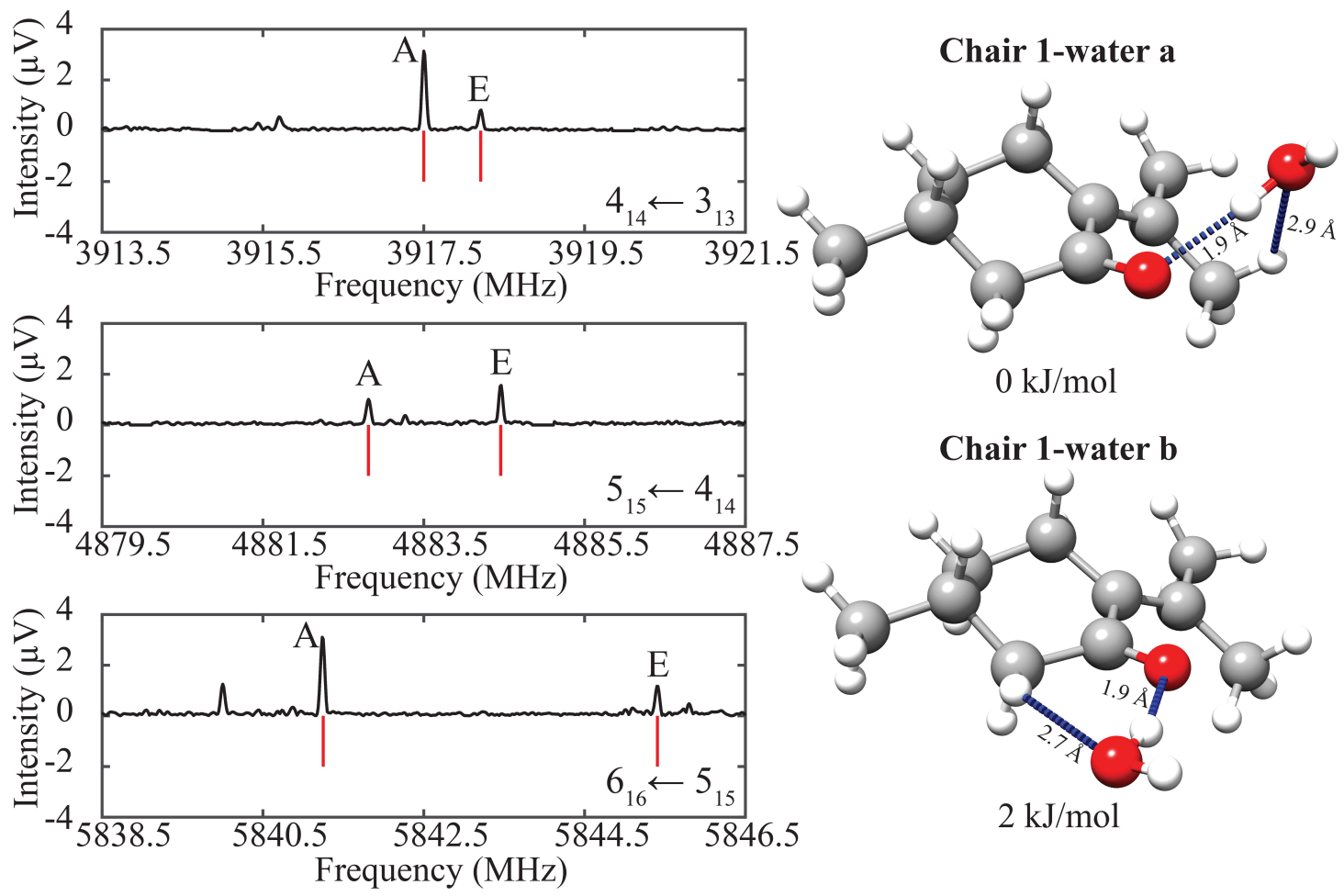

$0 \mathrm{~kJ} / \mathrm{mol}$

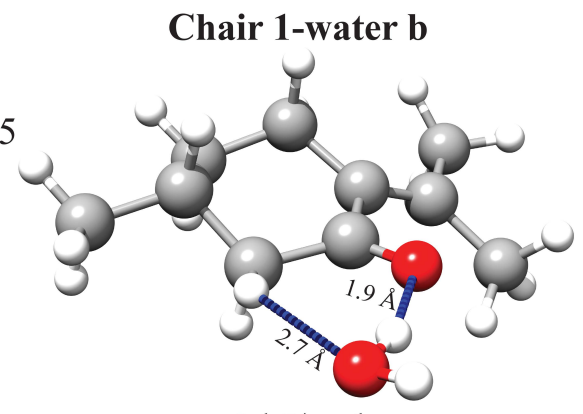

$2 \mathrm{~kJ} / \mathrm{mol}$

Figure 5 Parts of the broadband rotational spectrum ( 2 million acquisitions and using neon as carrier gas) of pulegone with water. The three sections are zoom-ins of individual rotational transitions to illustrate the doublet structure due to internal dynamics. In all parts, the upper traces (black) are the experimentally obtained spectrum. The lower (red) traces are stick spectra based on simulations using the fitted molecular constants for Chair 1-water a. The labeling of the rotational transitions follows the $J_{K_{a}} K_{c}$ scheme. On the right, two pulegone-water clusters calculated at the B3LYP-D3BJ/6-311++G(d,p) level of theory for the energetically lowest conformer of pulegone, Chair 1, are shown. The calculated hydrogen-bond distances are indicated.

the excess enantiomer of pulegone in the oil. Since it is known that essential oils contain (R)-pulegone almost in an enantiopure form, this will give us an opportunity to benchmark our technique for the analysis of complex chiral structures.

\subsection{The pulegone-water complex}

It is reported that pulegone is largely insoluble in water ${ }^{49}$, which is regarded as a major drawback in some applications. To overcome this, several strategies have been developed, such as supramolecular encapsulation ${ }^{50}$.

We are interested in understanding the interactions of a hydrophobic, insoluble molecule like pulegone with water at the molecular level. There are limited possibilities for water to interact with pulegone. The carbonyl group is the only polar group and can act as a hydrogen bond acceptor. To further explore this, geometry optimizations for different structures of the pulegone-water clusters (for the Chair 1 conformer) were performed at the M06-2X/6-311++G(d,p) and B3LYPD3BJ/6-311++G(d,p) levels of theory.
The two energetically most stable clusters, Chair 1-water a and Chair 1-water b, are represented in Figure 5. The hydrogen-bond interaction between the carbonyl group and water is present in both clusters. In addition, a secondary long range (dispersion) interaction between the oxygen of the water molecule and the hydrogens of the methyl top 2 can be expected.

One pulegone-water cluster was assigned in our experimental spectrum. The results for the calculated and experimentally determined molecular parameters for the Chair 1-water clusters $\mathrm{a}$ and $\mathrm{b}$ are presented in Table 2. Direct comparison between the calculated and experimental parameters allows us to assign the experimentally observed species to the energetically most stable Chair 1-water a cluster. Note that the B3LYP calculations with dispersion correction reproduce the experimentally determined values better than the M06-2X. The energy difference between the two clusters is small so that in principle we expect both clusters to be present in the spectrum. However, only Chair 1-water a was experimentally identified. This points to a low interconversion barrier between the two 
Table 2 Experimental and calculated molecular parameters for pulegone cluster with one water molecule (The experimental parameters of Chair 1-water a cluster were determined by fitting the internal rotation of methyl top 1 with XIAM). The observed rotational transitions are given using the following nomenclature: $\mathrm{P}$ brunch $\triangle \mathrm{J}=-1, \mathrm{Q}$ brunch $\triangle \mathrm{J}=0, \mathrm{R}$ brunch $\triangle \mathrm{J}=+1 ; \mathrm{a}, \mathrm{b}$, and c correspond to the respective type of the rotational transitions.

\begin{tabular}{|c|c|c|c|c|c|}
\hline \multirow[t]{2}{*}{ Parameter } & \multirow[t]{2}{*}{ Experimental } & \multicolumn{2}{|c|}{ M06-2X/6-311++G(d,p) } & \multicolumn{2}{|c|}{ B3LYP-D3BJ/6-311++G(d,p) } \\
\hline & & $\begin{array}{c}\text { Chair } 1-\text { water a } \\
1356.52\end{array}$ & $\begin{array}{c}\text { Chair 1-water b } \\
966.72\end{array}$ & $\begin{array}{c}\text { Chair 1-water a } \\
1295.96\end{array}$ & $\begin{array}{c}\text { Chair 1-water b } \\
940.30\end{array}$ \\
\hline $\mathrm{B} / \mathrm{MHz}$ & $565.42463(59)$ & 568.05 & 674.96 & 566.34 & 677.32 \\
\hline $\mathrm{C} / \mathrm{MHz}$ & $467.25183(44)$ & 471.16 & 416.76 & 459.45 & 412.01 \\
\hline$\Delta_{J} / \mathrm{kHz}$ & $0.0501(40)$ & 0.05 & 0.08 & 0.05 & 0.40 \\
\hline$\Delta_{J K} / \mathrm{kHz}$ & $0.519(23)$ & 0.41 & 0.14 & 0.38 & -0.77 \\
\hline$\Delta_{K} / \mathrm{kHz}$ & $-1.354(89)$ & 0.1 & 0.47 & 0.15 & 0.45 \\
\hline$\delta_{J} / \mathrm{kHz}$ & $0.0238(27)$ & -0.01 & 0.02 & -0.13 & 0.11 \\
\hline$V_{3} / \mathrm{kJmol}^{-1}$ & $2.01013(38)$ & $1.93^{\ddagger}$ & & & \\
\hline$\measuredangle \delta / \mathrm{rad}$ & 1.96917 (37) & 1.89 & - & - & - \\
\hline$\measuredangle \varepsilon / \mathrm{rad}$ & $2.86344(21)$ & 2.48 & - & - & - \\
\hline$D_{p i 2 J}{ }^{*} / \mathrm{MHz}$ & $-0.03842(72)$ & - & - & - & - \\
\hline$D_{p i 2 K}^{*} / \mathrm{MHz}$ & $0.280(18)$ & - & - & - & - \\
\hline$\mu_{\mathrm{a}} / \mathrm{D}$ & ${ }^{a} R$ & 2.6 & 2.5 & -2.6 & 2.9 \\
\hline$\mu_{\mathrm{b}} / \mathrm{D}$ & ${ }^{b} R,{ }^{b} Q$ & 1.7 & -2.6 & 1.8 & -2.9 \\
\hline$\mu_{\mathrm{b}} / \mathrm{D}$ & ${ }^{c} R$ & 0.3 & -0.2 & -0.5 & -0.3 \\
\hline $\begin{array}{l}\Delta \mathrm{E} / \mathrm{kJmol}^{-1} \\
\sigma^{\diamond} / \mathrm{kHz} \\
\mathrm{N}^{\dagger}\end{array}$ & $\begin{array}{c}9.83 \\
84\end{array}$ & 0 & 2.0 & 0 & 2.3 \\
\hline
\end{tabular}

clusters.

The secondary interaction of water with the methyl top 2 hinders its internal rotation, while internal rotation of methyl top 1 is still feasible. The calculated barrier for this internal rotation is $1.93 \mathrm{~kJ} / \mathrm{mol}$ and consequently low enough to give rise to internal rotation splitting of a single methyl group, resulting in doublets. This is in agreement with our experimental observations. An excerpt from the broadband spectrum of pulegone with water is presented in Figure 5, where the respective splittings due to the internal rotation of Chair 1 interacting with one water molecule are shown. The results for the internal rotation are summarized in Table 2 . The experimentally determined barrier height for the internal rotation is $2.01013(38) \mathrm{kJ} / \mathrm{mol}$, and thus de facto identical to the value for the pulegone monomer. This shows that methyl top 1 is indeed unaffacted by the addition of water.

\section{Conclusion and Outlook}

The internal dynamics of a molecule with two non-equivalent methyl tops was studied on the example of pulegone. Using $a b$ initio calculations (MP2/6-311++G(d,p) level of theory), we analyzed its four stable conformers. The broadband rotational spectrum reveals two conformers, including the previously reported lowest energy structure, and their rich internal dynamics due to the methyl tops attached to an $\mathrm{sp}^{2}$ hybridized carbon atom. The ${ }^{13} \mathrm{C}$-monosubstituted isotopologues in natural abundance were assigned, from which we obtained accurate structural information.

No spectroscopic data on the interaction of pulegone with water has been reported before. In the present work, we studied the interaction of the lowest energy conformer of pulegone with one water molecule to improve our knowledge of molecule-water interactions. Water locks the internal rotation of one of the methyl groups due to a secondary interaction, while the other methyl top remains unhindered in its internal rotation. The internal rotation of pulegone in the complex with water could be therefore analyzed as well. 
Finally, M3WM was worked out to differentiate the enantiomers of pulegone. The main advantages of using the rotational spectroscopy based technique are its fingerprint character regarding different molecular conformers and high resolution. M3WM is a nonlinear, coherent and resonant technique, which renders it a powerful method for enantiomeric differentiation in complex mixtures in the gas phase. An interesting next step is thus the chiral analysis in complex mixtures such as essential oils.

\section{Acknowledgement}

A. K. acknowledges the financial support of the IMPRSUFAST and the use of the GWDG computing cluster. C. P. acknowledges grant by the Alexander von Humboldt Stiftung.

M. M. Q. M. thanks the Universidad de Jaén for a predoctoral fellowship and the Max Planck Society and the Prince of Asturias Foundation for a Max Planck-Prince of Asturias Award Mobility Grant. The authors are also thankful to the Centro de Servicios de Informática y Redes de Comunicaciones (CSIRC), University of Granada (UGR), for computational time and facilities.

J. C. L, S. B., and P. P. acknowledge the Ministerio de Economía y Competitividad (Grant CTQ 2016-75253-P) for financial support. P. P. acknowledges the University of Valladolid for a mobility grant.

[1] T. G. Vale, F. J. A. Matos, T. C. M. de Lima and G. S. B. Viana, J. Ethnopharmacol., 1999, 67, 127-133.

[2] G. S. B. Viana, T. G. Vale, C. M. Silva and F. J. A. Matos, Biol. Pharm. Bull., 2000, 23, 1314-1317.

[3] J. Silva, W. Abebe, S. M. Sousa, V. G. Duarte, M. I. L. Machado and F. J. A. Matos, J. Ethnopharmacol., 2003, 89, 277-283.

[4] J. C. Brookes, A. P. Horsfield and A. M. Stoneham, J. R. Soc. Interface, $2009,6,75-86$.

[5] D. Schmitz, V. A. Shubert, B. M. Giuliano and M. Schnell, J. Chem. Phys., 2014, 141, 034304.

[6] C. Pérez, A. Krin, A. L. Steber, J. C. López, Z. Kisiel and M. Schnell, J. Phys. Chem. Lett., 2016, 7, 154-160.

[7] Z. Kisiel, O. Desyatnyk, E. Białkowska-Jaworska and L. Pszczółkowski, Phys. Chem. Chem. Phys., 2003, 5, 820-826.

[8] J. R. Avilés Moreno, T. R. Huet and J. J. López González, Struct. Chem., 2013, 24, 1163-1170.

[9] D. Schmitz, V. A. Shubert, T. Betz and M. Schnell, Front. Chem., 2015, 3, $1-15$.

[10] V. A. Shubert, D. Schmitz, C. Medcraft, A. Krin, D. Patterson, J. M. Doyle and M. Schnell, J. Chem. Phys., 2015, 142, 214201.

[11] S. R. Domingos, C. Pérez, C. Medcraft, P. Pinacho and M. Schnell, Phys. Chem. Chem. Phys., 2016, 18, 16682-16689.

[12] B. M. Mitzner, E. T. Theimer and S. K. Freeman, Appl. Spectrosc., 1965, 19, 1969-1985.

[13] K. R. Strehle, P. Rösch, D. Berg, H. Schulz and J. Popp, J. Agric. Food. Chem., 2006, 54, 7020-7026.

[14] C. Guo, R. D. Shah, R. Dukor, T. B. Freedman, X. Cao and L. Nafie, Vib. Spectrosc., 2006, 42, 254-272.

[15] G. Longhi, S. Abbate, R. Gangemi, E. Giorgio and C. Rosini, J. Phys. Chem. A., 2006, 110, 4958-4968.
[16] F. Ureña, J. R. Avilés Moreno, and J. J. López González, Tetrahedron: Asymmetry, 2009, 20, 89-97.

[17] J. Kraitchman, Am. J. Phys., 1953, 17, 17-24.

[18] T. Mizutani, T. Ema and H. Ogoshi, Tetrahedron, 1995, 51, 473 - 484.

[19] S. Blanco, P. Pinacho and J. C. López, Angew. Chem. Int. Ed. Engl., 2016, 55, 9331-9335.

[20] C. Pérez, J. C. López, S. Blanco and M. Schnell, J. Phys. Chem. Lett., 2016, 7, 4053-4058.

[21] D. Patterson, M. Schnell and J. M. Doyle, Nature, 2013, 497, 475-478.

[22] D. Patterson and J. M. Doyle, Phys. Rev. Lett., 2013, 111, 75-86.

[23] V. A. Shubert, D. Schmitz and M. Schnell, J. Mol. Spectrosc., 2014, 300, 31-36.

[24] S. Eibenberger, J. Doyle and D. Patterson, Phys. Rev. Lett., 2017, 118, 123002.

[25] C. Pérez, A. L. Steber, S. R. Domingos, A. Krin, D. Schmitz and M. Schnell, Angew. Chem. Int. Ed. Engl., 10.1002/anie201704901.

[26] Handbook of ESSENTIAL OILS. Science,Technology and Applications., ed. K. H. Can Başer and G. Buchbauer, CRC Press, 2010.

[27] R. H. McClanahan, D. Thomassen, J. T. Slattery and S. D. Nelson, Chem. Res. Toxicol., 1989, 2, 349-355.

[28] J. A. Bakerink, S. M. Gospe, R. J. Dimand and M. W. Eldridge, Pediatrics, 1996, 98, 944-947.

[29] W. Engel, J. Agric. Food Chem., 2003, 51, 6589-6597.

[30] W. P. Gordon, A. C. Huitric, C. L. Seth, R. H. McClanahan and S. D. Nelson, Drug Metab. Dispos., 1987, 15, 589-594.

[31] J. R. Avilés-Moreno, E. Ureña Horno, F. Partal Ureña and J. J. López González, Spectrochim. Acta A Mol. Biomol. Spectrosc., 2011, 79, 767-776.

[32] D. Schmitz, V. A. Shubert, T. Betz and M. Schnell, J. Mol. Spectrosc., 2012, 280, 77-84.

[33] C. Pérez, S. Lobsiger, N. A. Seifert, D. P. Zaleski, B. Temelso, G. C. Shields, Z. Kisiel and B. H. Pate, Chem. Phys. Lett., 2013, 571, 1-15.

[34] D. Plusquellic, JB95, available at http://www.nist.gov/pml/electromagnetics/grp05/jb95.cfm.

[35] C. M. Western, J. Quant. Spectrosc. Radiat. Transfer, 2016, 186, 221242.

[36] H. M. Pickett, J. Mol. Spectrosc., 1991, 148, 371-377.

[37] H. Hartwig and H. Dreizler, Z. Naturforsch, 1996, 51a, 923-932.

[38] R. C. Woods, J. Mol. Spectrosc., 1966, 21, 4-24.

[39] V. A. Shubert, D. Schmitz, D. Patterson, J. M. Doyle and M. Schnell, Angew. Chem. Int. Ed. Engl., 2014, 53, 1152-1155.

[40] V. A. Shubert, D. Schmitz, C. Pérez, C. Medcraft, A. Krin, S. R. Domingos and M. Schnell, J. Phys. Chem. Lett, 2016, 7, 341-350.

[41] M. J. Frisch, G. W. Trucks, H. B. Schlegel, G. E. Scuseria, M. A. Robb, J. R. Cheeseman, G. Scalmani, V. Barone, B. Mennucci, G. A. Petersson, H. Nakatsuji, M. Caricato, X. Li, H. P. Hratchian, A. F. Izmaylov, J. Bloino, G. Zheng, J. L. Sonnenberg, M. Hada, M. Ehara, K. Toyota, R. Fukuda, J. Hasegawa, M. Ishida, T. Nakajima, Y. Honda, O. Kitao, H. Nakai, T. Vreven, J. A. Montgomery, Jr., J. E. Peralta, F. Ogliaro, M. Bearpark, J. J. Heyd, E. Brothers, K. N. Kudin, V. N. Staroverov, R. Kobayashi, J. Normand, K. Raghavachari, A. Rendell, J. C. Burant, S. S. Iyengar, J. Tomasi, M. Cossi, N. Rega, J. M. Millam, M. Klene, J. E. Knox, J. B. Cross, V. Bakken, C. Adamo, J. Jaramillo, R. Gomperts, R. E. Stratmann, O. Yazyev, A. J. Austin, R. Cammi, C. Pomelli, J. W. Ochterski, R. L. Martin, K. Morokuma, V. G. Zakrzewski, G. A. Voth, P. Salvador, J. J. Dannenberg, S. Dapprich, A. D. Daniels, O. Farkas, J. B. Foresman, J. V. Ortiz, J. Cioslowski and D. J. Fox, Gaussian 09 Revision E.01, Gaussian Inc. Wallingford CT 2009.

[42] Z. Kisiel, PROSPE Programs for ROtational SPEctroscopy, available at www.ifpan.edu.pl/ kisiel/prospe.htm.

[43] C. C. Costain, Trans. Am. Crystallogr. Assoc., 1966, 2, 157-164.

[44] C. Medcraft and M. Schnell, Z. Phys. Chem., 2015, 230, 1-14. 
[45] J. M. Vacherand, B. P. Van Eijck, J. Burie and J. Demaison, J. Mol. Spectrosc., 1986, 118, 355-362.

[46] H. Hartwig, XIAM Manual, 1996, available at http://www.ifpan.edu.pl/ kisiel/introt/xiam/xiam-v25.txt.

[47] S. Lobsiger, C. Pérez, L. Evangelisti, K. K. Lehmann and B. H. Pate, J. Phys. Chem. Lett, 2015, 6, 196-200.

[48] D. Schmitz, V. A. Shubert, D. Patterson, A. Krin and M. Schnell, J. Phys. Chem. Lett, 2015, 6, 1493-1498.

[49] The Merck Index - An Encyclopedia of Chemicals, Drugs, and Biologicals, ed. M. O'Neil, RSC Publishing, 2013, p. 1470.

[50] T.-W. Moon, J. W. Lee, K.-H. Jhee, K.-W. Khang, H.-S. Jeong, S.-A. Yang and H.-J. Kim, Bull. Korean. Chem. Soc., 2008, 29, 1579-1582. 